\title{
MACHINE LEARNING-BASED EFFICIENT CLUSTERING AND IMPROVE QUALITY OF SERVICE IN MANET
}

\author{
V. Surya Narayana Reddy \\ Research Scholar, Department of Information Science and Engineering, Visvesvaraya Technological \\ University, Belagavi. Nagarjuna College of Engineering and Technology, Research Centre, \\ Bangalore, Karnataka, India, suryanarayananphd290@gmail.com

\section{Dr. Jitendranath Mungara}

Principal \& Prof., Department of Computer Science and Engineering, Nagarjuna College of Engineering and Technology, Bangalore, Karnataka, India.

\begin{abstract}
A Mobile Ad hoc Network (MANET) is a self-governing system in that numerous nodes are connected with each other, applying multi-hop wireless links. MANET has overturned society owing to their infrastructure-less decentralized modes of transaction. As a result, researchers have concentrated on discovering better directions to operate the probability of MANETs completely. The current initiation of machine learning methods has prepared it to concern artificial intelligence to increase the best approaches for this function. In this work, Machine Learning-based Efficient Clustering and Improve Quality of Service in MANET (MLEC) is proposed. In this work, we improve the clustering technique that applied Quality of Service (QoS) parameters to choose Cluster Heads (CHs). The cluster formation and $\mathrm{CH}$ selection is accessible via a multi-objective fitness function applying Particle Swarm Optimization (PSO) algorithm. This approach gives three inputs: node mobility, energy utilization rate, and bandwidth. The algorithm establishes a higher rate of achievement in forecasting output values also could correctly recognize better $\mathrm{CH}$ that should offer the well-organized routing. In addition, it reduces the intra-cluster distance among nodes and their relevant CHs. The proposed work is experimented with broadly in the network simulator and compared with the other baseline protocols.
\end{abstract}

Keywords: Machine learning; particle swarm optimization algorithm; clustering; quality of service improvement; mobile ad hoc network.

\section{Introduction}

A MANET is a wireless multi-hop network that contains mobile nodes which can travel autonomously. MANET has a lacking structure since the nodes travel randomly. This topology can repeatedly adopt, which is not need any access points for communication [Wang et al, 2014]. In MANET, mobile nodes can transmit with every other frankly otherwise intermediate. The source node can transmit with the other node openly inadequate radio propagation, also ultimately via multi-hop routing with all other nodes. To assist this type of transmission, several routing techniques have previously been formulated. However, these routing protocols increase the routing overhead, energy utilization rate and raise the transmission delay. ML is examining how the computer reproduces otherwise comprehends the learn human being behavior [Xue and Zhu, 2009]. The objective is to receive the recent knowledge otherwise the ability, categorize the knowledge structure that can build reformist enhancement of its possess function. It is the interior of Artificial Intelligence (AI); It is a basic method that facilitates to have the aptitude; the application of it is attained in several fields of AI. Also, it is mostly applying the mode of induction as well as the deduction but not the subtraction.

Together with the growth of Internet technology also multimedia technical, AI study has egresses an amount of new problems. AI has been concerned enhance concentration in several fields that is a border of fields that is applied to reproduce human thought [Rupali and Amit, 2017]. AI is an intelligence that is majorly smarter than the greatest human brain in basically each field, plus linguistic logic and computer science. It is related with essential also most significant views in the life. The Machine Learning investigation demonstrates the computation model otherwise, the understanding model, along with the learning approach of humanity via the functioning, the cognitive science, formulates each type of learning theory and examines the general technique. 
Clustering is a significant method that resolves several issues of MANET; also it enhances the lifespan and provides network scalability. Here better performance nodes are select the $\mathrm{CH}$, and these $\mathrm{CHs}$ are joined; the mobile nodes are formed into virtual groups is known as clusters [Mehrkanoon et al, 2014]. In addition, clusterbased route is used to enhance the network management and minimizes the nodes routing table. Though, $\mathrm{CHs}$ tolerate the additional work load of communication. Hence, $\mathrm{CH}$ energy is depleted earlier, and the death of CHs that eventually division also disgrace the network lifetime. Furthermore, the node mobility is the main reason for link failure. A Self-organization-based Bio-inspired Clustering Approach (SBCA) provides better stability because of its zone-based node exploitation. In this approach, the $\mathrm{CH}$ is elected via a bio-inspired method that provides more efficiency. This approach minimizes congestion and enhances network function, and minimizes energy utilization. However, this approach increases the routing overhead, and it can't balance the network load. So, this approach minimized the entire network performance [Aftab et al, 2017]. To solve these problems, Machine Learning-based Efficient Clustering and Improve QoS is proposed. Here, clustering technique that applied QoS parameters to choose $\mathrm{CHs}$. The cluster formation and $\mathrm{CH}$ selection process is established through the PSO algorithm. Node mobility, energy utilization rate, and bandwidth parameters are used for compute the PSO fitness function. In addition, it reduces the intra-cluster distance among nodes and their relevant CHs. Therefore, designing an efficient clustering algorithm with minimum overhead becomes a good approach to increasing the network lifetime of the MANET.

This article is structured as follows: Section 2 describes the related research work regarding machine learning clustering approach. Section 3 explains the Machine Learning-based Efficient Clustering and Improve QoS. Section 4 contains simulation results of MLEC and SBCA approaches. Finally section 5 present the conclusion.

\section{Related Work}

Stabilized Clustering approach to enhance the stability of cluster formation also improves efficiency. This approach decides the CH through QoS standard by the Moth Flame Optimization method. In this approach, assistant $\mathrm{CH}$ aids to avoid the $\mathrm{CH}$ weakening; thus, it runs effectively [Wang and Qing, 2010]. QoS-guided Dynamic Scheduling approach is to recognize the best placements for all types of tasks; also, it schedules the clusters. It is a lightweight approach and provides a QoS assurance [Alowish et al, 2020]. QoS-oriented scheduling and auto-scaling approach is used to schedule jobs in the cluster. This approach concentrates on the significant QoS requirement. This inescapability is applied to forecast whether a job can be talented before its target also guesses suitable resource formation. However, this approach can't satisfy the QoS services [Chen et al, 2019].

Multi Dimension-Affinity Propagation Clustering approach is applying the ML to decide the graininess. This approach attains a sufficient stage of forecast correctness with lesser training data via a discovered forecast operation by the key standards [Xing et al, 2019]. Support Vector Machine method is applying a heuristic scheduling rule through the status of resource also job deadline. Denoising auto-encoder with fuzzy clustering approach concentrates on utilizes context information also improves over fitting. The fuzzy clustering technique overcomes the desert that the function is simple to be affected through the amount of clusters. However, this approach not predicts the QoS accurately [Koshimizu et al, 2020].

Machine intelligence is working as pre-processing to improve the present time series examples. The Fuzzy-CMeans clustering also the weight exponential model for enhancing optimum results and effectively improves the prediction accuracy. A suitable time series prediction model assisted by appropriate machine intelligence has been established [Yin et al, 2020]. Artificial neural networks established ML techniques may be applied for resolving several network issues. This approach comprises periodic spiking, also deep neural networks that are relevant to applications [Aldhyani et al, 2020]. An unsupervised univariate filter feature selection procedure is utilized to diminish the ill effects of clustering operation [Nalluri and Kurra, 2021].

An energy-aware multipath routing approach is using a PSO technique to solve optimization issues. This technique provides optimal loop-free routes that solve link disjoint routes. This approach evaluates the parameters like energy, optimal traffic ratio among sender and receiver, communication cost that enhance the routing function [Robinson and Rajaram, 2015]. The clustering Hierarchy framework provides better link quality. PSO method is used to choose the optimized attributes for selecting the better performance. PSO method is used for enhancing the lifespan. This method provides better function and minimizes energy utilization [Trivedi and Sharma, 2016].

Link lifetime prediction approach using PSO technique for recover the route in the network. It forecast the link lifespan and measuring the bandwidth. Here, the fuzzy optimization method is to check the node status. During data communication, every node checks the node status. PSO technique reduces both the packet loss and routing overhead [Zhou et al, 2016]. BatFuzzyBee approach that utilize position information to handle congestion. cluster-based network formation is utilized to minimize the loss of packet as well as energy saving. BatFuzzyBee can discover the reliable route, while the has nodes with dissimilar communication ranges with the least energy utilization [Suchitha and Prasanthi, 2021]. The position-based QoS routing approach employs bandwidth reservation for revealed paths from sender to receiver. An Adaptive multi-QoS cooperative routing 
approach improves the routing function. This approach selects the better transmission node through multiple QoS. This selects the node form the better route from sender to receiver [Abdrabou and Zhuang, 2006].

Cluster distance-based data forwarding and optimal $\mathrm{CH}$ selection applying fuzzy inference algorithm. Here, builds the cluster then the major $\mathrm{CH}$ as well as helper $\mathrm{CHs}$ are elected. Suppose, while the main leader is disjointed, the helper cluster perform to avoid network loss. Since the communication ranges is different sizes. These cluster sets are build in a fuzzy inference manner, hence increases the lifespan and minimizes the hotspot problem [Joshi and Biradar, 2021]. Integrated Cross Layer optimization approach that improve the QoS. It is solved through various optimization model such as optimization of queueing model, obstacle aware interference modelling, channel modelling, video distortion model [Chavan and Reddy, 2021].

\section{MACHINE LEARNING-BASED EFFICIENT CLUSTERING AND IMPROVE QUALITY}

The main aim of this approach is used to improve the QoS service and enhance the network lifespan. Unequal allocation of $\mathrm{CH}$ loads ensures mobile node energy utilization is earlier; as a result, $\mathrm{CH}$ dead. It makes a link failure; hence choose another $\mathrm{CH}$ otherwise re-clustering. Thus, we have considered $\mathrm{CH}$ strength as an essential factor. Here, initially selects the $\mathrm{CH}$ by mobile node bandwidth, mobility, and energy utilization rate. The node with has the highest bandwidth, node degree, and lowest mobility, energy utilization rate, distance is selected as a CH. Fig. 1 explains the example diagram of the MLEC Approach.

This Here, every mobile node broadcasts the HELLO message among one-hop neighbors. This HELLO message contains the NM, NEU, and NB. All one-hop neighbor nodes receive the HELLO message. Every mobile node selects the CH based on NM, MEU, and NB using the PSO technique. Node Mobility extends to additional $\mathrm{CH}$ re-selection, also updating the link that marks in worst cluster stability. According to consider the node mobility is a significant factor. Here, compute the node mobility (NM) through the received signal strength of the node among two consecutive HELLO packets that is in reverse proportional to the distance between the source and destination. It is computed by the formula is given below.

$$
\mathrm{NM}_{\mathrm{i} \rightarrow \mathrm{j}}=10 \log _{10} \frac{\text { New RSS HP }}{\text { Old RSS HP }}
$$

here, new New RSS HP ${ }_{i \rightarrow j}$ denotes the new RSS HELLO packets and Old RSS HP ${ }_{i \rightarrow j}$ if new New RSS HP ${ }_{i \rightarrow j}>$ Old RSS HP ${ }_{i \rightarrow j}$ represents the node movement is positive, that is, the node $j$ is closer to the node $i$, and New RSS HP ${ }_{i \rightarrow j}<$ Old RSS HP $_{i \rightarrow j}$ represents the node $j$ is moving from the node $i$.

In MANET, the node Energy utilization (NEU) is a significant factor. At time interval $\Delta_{t}$ the Energy utilization of a node $n_{i}$ is given below.

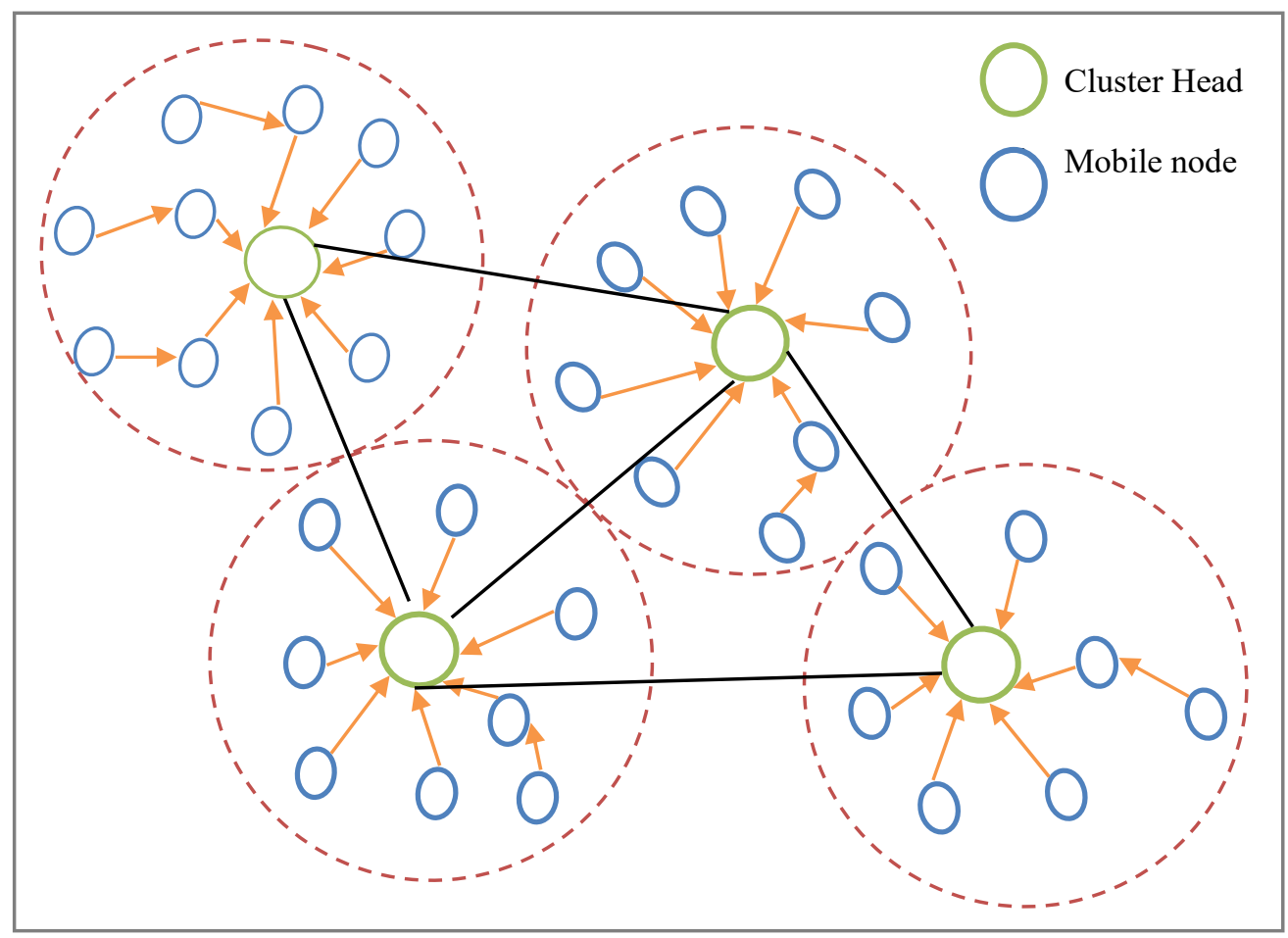

Fig. 1. Architecture of REDC 


$$
\mathrm{NEU}_{(\Delta t, \mathrm{i})}=\mathrm{EN}_{\text {utilization }(\mathrm{i}, \mathrm{t})}-\mathrm{EN}_{\text {utilization }\left(\mathrm{i}, \mathrm{t}_{1}\right)}
$$

Here, indicates the difference among utilized energy of $i^{\text {th }}$ node $i$ at time $t$ as well as time $t 1$, correspondingly. Node Bandwidth (NB) computation is a significant factor in balancing the network load.

$$
\begin{aligned}
& \mathrm{NB}=\frac{\text { Chann }_{\text {Bandwidth }}-\text { utilized }_{\text {Bandwidth }}}{\text { Weight Feature }} \\
& \text { Weight Feature }=\frac{\text { RO }+ \text { PS }}{\text { PS }}
\end{aligned}
$$

Here, RO denotes the routing overhead, and PS represents the packet size in the network. In MANET, wellstructured clusters also a significant factor that is leading how nodes are associated with a $\mathrm{CH}$ that forms a cluster. After, collection of CHs, we build the cluster using the PSO method. This method launches the particle swarm, and the fitness function is estimated by the node location and node velocity.

Particle Swarm Optimization: PSO is a current random search algorithm that employs the notion of social communication to solve the problem. It applies an amount of particles which make up a pre-set swarm (group) $N p$, moving around in the network space d expecting the best solution. Every particle $P i, 1 \leq i \leq N$ denotes a possible solution has a fitness value that is measured through the fitness function and have a location $\mathrm{L} i, d_{i}, 1 \leq d$ $\leq D$, and velocity of particles represents the $V_{i, d}$ is handled as a point in $D$ space that adapts its pb and gb. Here, pbest $(\mathrm{pb})$ represents the location of own past best also gbest $(\mathrm{gb})$ represents the location of the whole swarm.

The procedures of the PSO algorithm is given below.

Every particle attempts to adapt its location applying the following information also every iteration that modifies its location and velocity utilizing the formulas given below.

(i) The present location

(ii) The present velocity

(iii) The distance among the present location and $\mathrm{Pbi}$

(iv) The distance among the present location and $\mathrm{Gb}$.

$$
\mathrm{L}_{\mathrm{i}, \mathrm{d}}(\mathrm{t}+1)=\mathrm{L}_{\mathrm{i}, \mathrm{d}}(\mathrm{t})+\mathrm{V}_{\mathrm{i}, \mathrm{d}}(\mathrm{t})
$$

$$
\begin{aligned}
& \mathrm{V}_{\mathrm{i}, \mathrm{d}}=\beta * \mathrm{~V}_{\mathrm{i}, \mathrm{d}}(\mathrm{t})+\lambda_{1} * \mathrm{R}_{1} *\left(\mathrm{Lpb}_{\mathrm{i}, \mathrm{d}}-\mathrm{L}_{\mathrm{i}, \mathrm{d}}(\mathrm{t})\right)+ \\
& \lambda_{2} * \mathrm{R}_{2} *\left(\mathrm{Lgb}_{\mathrm{d}}-\mathrm{L}_{\mathrm{i}, \mathrm{d}}(\mathrm{t})\right)
\end{aligned}
$$

Here, $\beta$ denotes the weight, $\lambda_{1}$ as well as $\lambda_{2}$ denote an invariables for acceleration factors, and $\mathrm{R}_{1}$ as well as $\mathrm{R}_{2}$ indicate the random numbers among 0 and 1 . The iteration goes on until some termination condition, that is, a fixed number of iterations or adequate Gbest value, is obtained.

\section{PSO based Route Formation:}

In this approach, the PSO technique is select the $\mathrm{CH}$ by node mobility, node energy utilization and bandwidth. It is explained in algorithm below.

1: Initiate the each particle $p_{i}$ location $\left(L_{i}\right)$ and velocity $\left(V_{i}\right)$

2: Compute the fitness value of every particle also initiates $\mathrm{Pb}$ and $\mathrm{gp}$

3: Do iteration $i=1$ to $n\{$

4: Modify velocity and location of particle

5: Measure the Pi fitness $F(p i)\{$

$\mathrm{F}(\mathrm{Pi})=\left(\lambda_{1} * \mathrm{NM}\right)+\left(\lambda_{2} * \mathrm{NE}\right)+\left(\lambda_{3} * \mathrm{NB}\right)$

6:\} If $F\left(p_{i}\right)>F\left(P b_{i}\right)\{$

7: Modifiy $P b_{i}$ explicitly $P b_{i}=p_{i}$

8: $\}$ If $F(P b)>F(G b)\{$

9: Modify $G$ b explicitly $G b_{i}=P b_{i}$

10: \}if Finish all iteration \{

11: $\}$ end

12: Else go to step 4

13: $\}\}$

Hence, the position and velocity of the particles are governed by its present velocity, its self progression factor, and the social interaction. PSO technique is optimizing the $\mathrm{CH}$ strength and attains the node stability in MANET.

While increases the distance among the $\mathrm{CH}$ with corresponding cluster member nodes, they necessitate draining additional energy to transmit with their CHs. Hence, such nodes expire rapidly, as a result decreases the network lifespan. Accordingly, in intra-cluster communication we select the relay node to the closer $\mathrm{CH}$ from the set of 
CHs inside the communication range. Therefore, diminish the energy utilization for intra-cluster communication. The computation of average distance is given below.

Avg distance $=\left(\sum_{\mathrm{i}=1}^{\mathrm{h}} \operatorname{dis} \operatorname{tance}\left(\mathrm{n}_{\mathrm{i}}, \mathrm{CH}\right)\right) / \mathrm{h}$

dis tance $\left(\mathrm{n}_{\mathrm{i}}, \mathrm{CH}\right)$ represents the distance among the node and the $\mathrm{CH}, \mathrm{h}$ denotes the nodes of a communication range CHs. In addition, the decrease of signal strength is a significant feature that is frankly relative to the distance among the source and destination. The power utilization rate is computed by the formula given below. $\mathrm{NS}_{\mathrm{Rec}}(\mathrm{d})=\mathrm{S}_{\text {Trans }} / \mathrm{d} \gamma$

Here, $\mathrm{NS}_{\mathrm{Rec}}(\mathrm{d})$ represents the received signal amplitude starting a source at a d distance from the destination, denotes the transmitted signal strength, represents the distance among the source and destination as well as $\gamma$ denotes the path loss factor. After form the clusters, if the source desires to transmit the data to a destination via PSO approach based to select the route. Every node updates its velocity and position does the iteration, and the resolution proceeds forward towards the best feasible result. The iteration extends, and every time the particle's present fitness value is compared with its own best value. On attaining better fitness, the Pbi, is substituted through the present value. Furthermore, the $\mathrm{Gb}$, is modified on getting the gb fitness value. Thus, improves the lifetime and minimizes the delay in the network.

\section{Network Simulation}

In this approach, we examine the function of the MLEC by applying the NS2 simulator. We measure the working performance of MLEC by equating SBCA results. The topology of the network is $500 \mathrm{~m} \times 500 \mathrm{metre}^{2}$ and disseminated with 150 nodes. The Constant Bit Rate traffic model is used for data packet transmission; also, the node packet size is 1024 bytes.

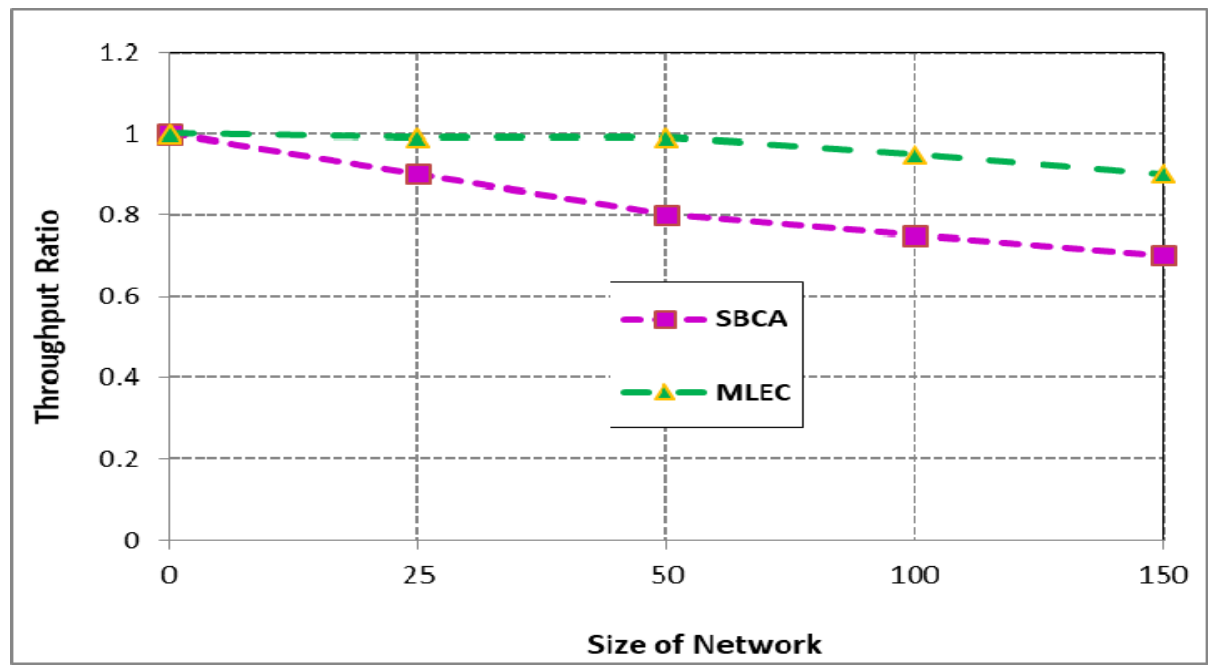

Fig. 2. Throughput Ratio of SBCA and MLEC Approach.

Our proposed MLEC approach attains better throughput efficiency than the SBEC approach is shown in Fig. 2. We observe that the MLEC approach increases the network throughput till 100 nodes then slightly decreased. Though, the SBCA approach function is degrading when increasing the network size owing to the retransmitting lost packets makes the additional delay. Also, this approach $\mathrm{CHs}$ are easily dead since it transmits the data packet to long-distance nodes. Furthermore, the CHs are can't balance the network load. But MLEC approach solves these problems, as a result, increases the network throughput. 


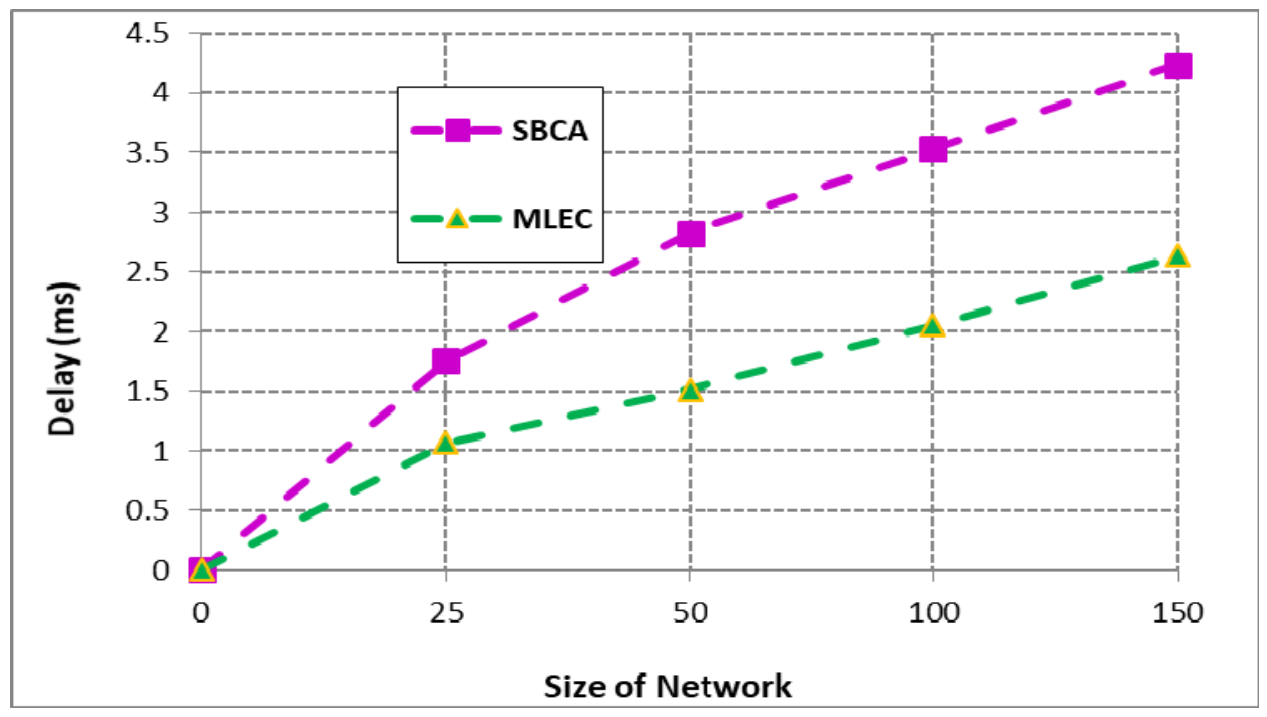

Fig. 3. Delay of DATA and REDC approach.

MLEC has a lesser delay and outperforms than the SBCA approach since the MLEC approach established the route by PSO that is minimized the intra-cluster distance; also, it selects the $\mathrm{CH}$ performs well. From this Fig. 3, the node density raises the MLEC approach is slightly increases the delay, but the SBCA approach heavily raises the delay since it can't use the optimal route from the source to destination.

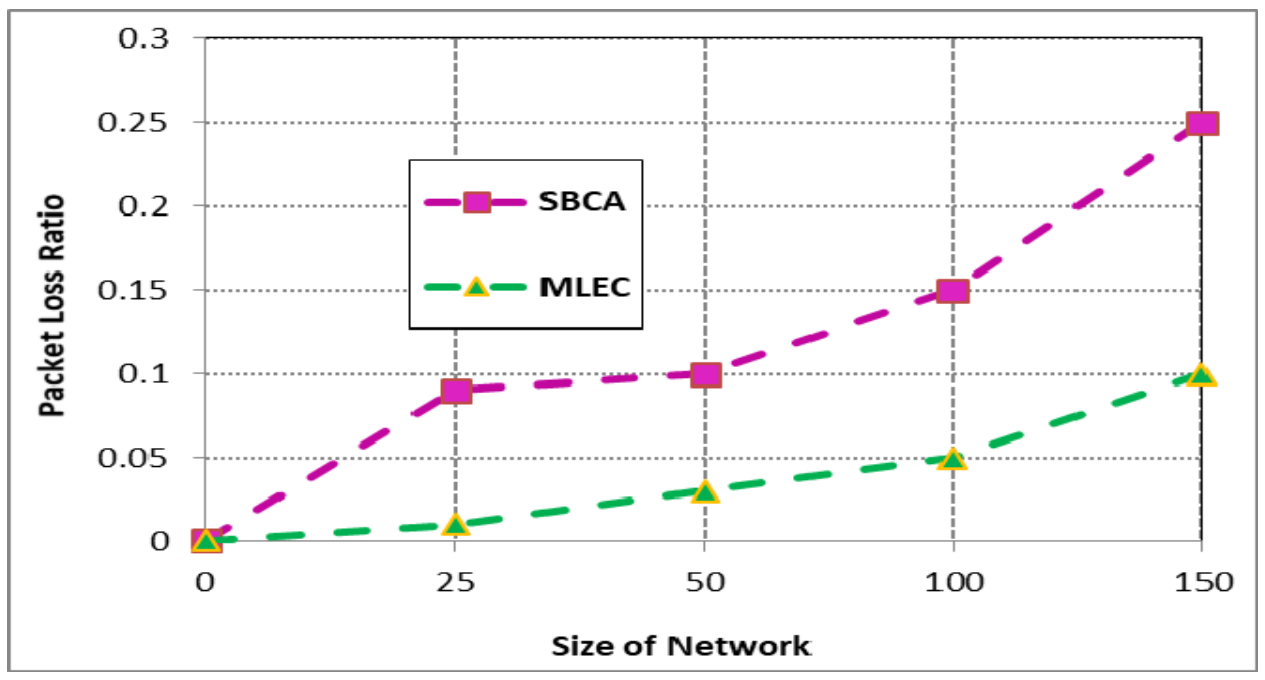

Fig. 4. Delay of DATA and REDC approach.

Fig. 4 explains the packet loss ratio of SBCA and MLEC that is one of the significant QoS parameters. When increases the network size, the SBCA approach loss of packet ratio is enhances significantly owing to the disturbances and collisions in the intra-cluster communication and inter-cluster communication. Conversely, the MLEC approach slightly increases the packet loss ratio when increases the network size. Regarding the residual energy consumption, our MLEC approach has great residual energy compared to the SBCA approach, as revealed in Fig 5 based on network size. In MLEC, select the CH by node bandwidth, utilized energy, and node mobility through the PSO technique. Also, the MLEC approach minimized the intra-cluster communication distance. Hence, the MLEC approach is minimized the more energy utilization in the network. But, the SBCA approach mainly concentrates only on node position. Thus, the SBCA approach has lesser residual energy than the MLEC approach. 


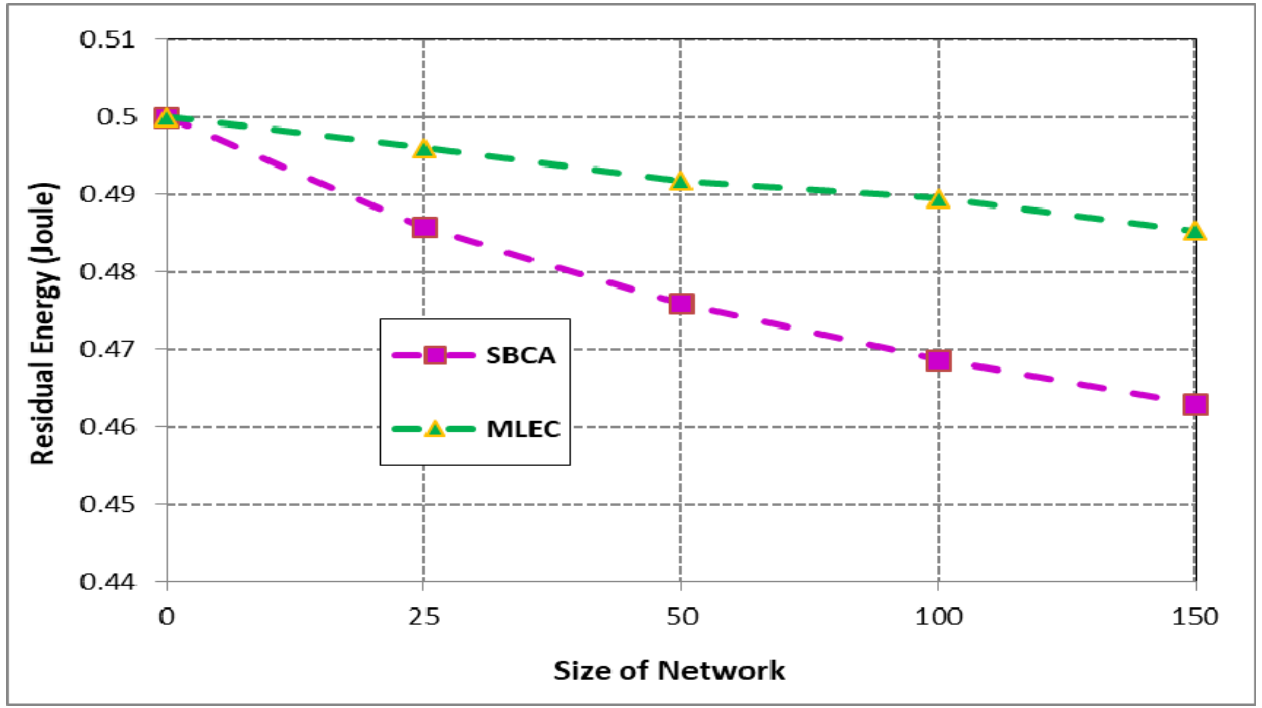

Fig. 5. Residual Energy of SBCA and MLEC Approach.

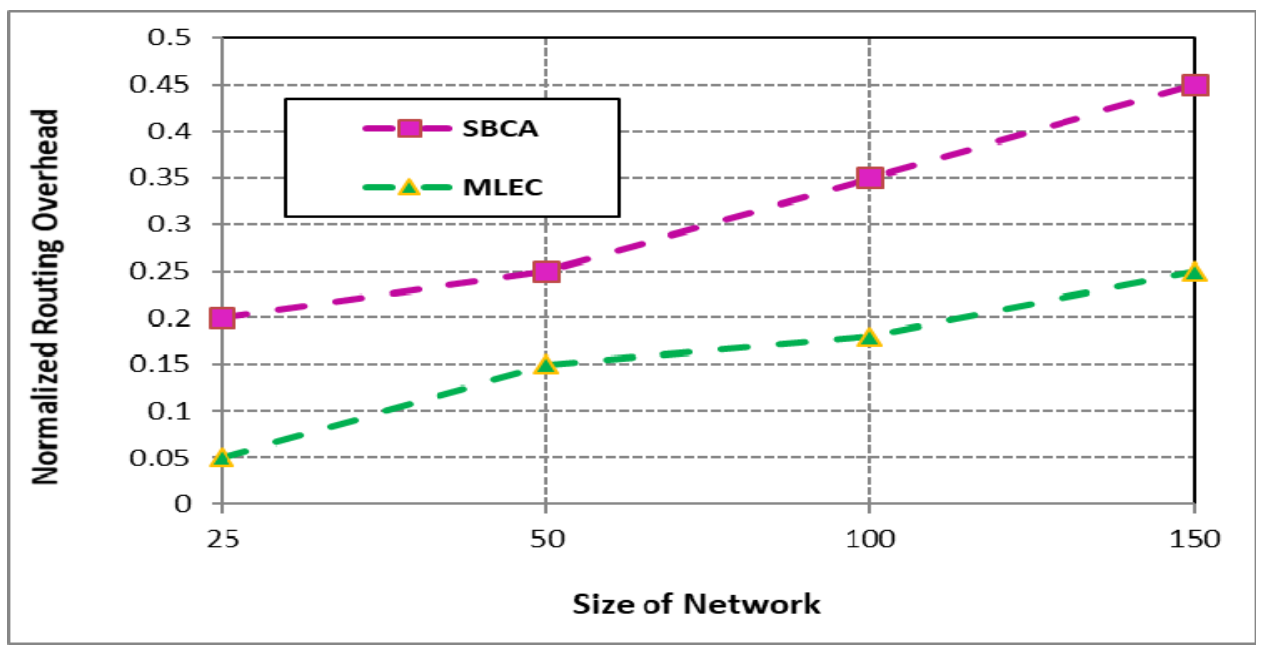

Fig. 6. Normalized Routing Overhead of SBCA and MLEC Approach.

Fig. 6 shows the normalized routing overheads of MLEC and SBCA approaches based on the network size. We noticed from fig 6, the overhead of the MLEC approach has a lesser overhead than the SBCA approach. The size of the network is increasing the SBCA approach is also raises the routing overhead since it increases the retransmit the loss of packets and packet collision. But, the MLEC approach raises the network size, the routing overhead is little increases but performs well than the SBCA approach.

\section{Conclusion}

In this paper, Machine Learning-based Efficient Clustering and Improve QoS in MANET is proposed. In this work, the PSO technique is used to form the clusters, and $\mathrm{CH}$ selection is accessible via a multi-objective fitness function. This approach gives three inputs: nodes mobility, energy utilization rate, and bandwidth parameters are give the input of the PSO, and it attained the best fitness value that correctly recognized the better $\mathrm{CH}$ that should offer the well-organized routing. Additionally, it reduces the intra-cluster distance among nodes and their relevant CHs. Simulation results illustrated that the MLEC approach is improved the throughput and residual energy in the network. Also, this approach minimized the delay and routing overhead, thus improved the QoS in the network.

\section{References}

[1] Wang, C.; Ye, B.; Wang, X.; Guo, S.; Lu, S. (2014): Delay and Capacity Analysis in MANETs with Correlated Mobility and \$ff $\$$ Cast Relay. IEEE Transactions on Parallel and Distributed Systems, 25(11), pp.2829-2839.

[2] Xue, M.; Zhu, C. (2009): A study and application on machine learning of artificial intelligence. In 2009 International Joint Conference on Artificial Intelligence (pp. 272-274). IEEE. 
[3] Rupali, M.; Amit, P. (2017): A review paper on general concepts of artificial intelligence and machine learning. International Advanced Research Journal in Science, Engineering and Technology, 4(4), pp. 79-82.

[4] Mehrkanoon, S.; Alzate, C.; Mall, R.; Langone, R.; Suykens, J. A. (2014): Multiclass semisupervised learning based upon kernel spectral clustering. IEEE transactions on neural networks and learning systems, 26(4), pp.720-733.

[5] Aftab, F., Zhang, Z., \& Ahmad, A. (2017). Self-organization based clustering in MANETs, using zone based group mobility. IEEE Access, 5, 27464-27476.

[6] Wang, L.; Qing, Y. (2010): The Study of Classification with SVM in MANeT. In 2010 International Conference on Measuring Technology and Mechatronics Automation, 3, pp. 994-996. IEEE.

[7] Alowish, M.; Shiraishi, Y.; Takano, Y.; Mohri, M.; Morii, M. (2020): Stabilized Clustering Enabled V2V Communication in an NDNSDVN Environment for Content Retrieval. IEEE Access, 8, pp.135138-135151.

[8] Chen, Z.; Luo, L.; Yang, H.; Yu, J.; Wen, M.; Zhang, C. (2019): GENIE: QoS-guided dynamic scheduling for CNN-based tasks on SME clusters. In 2019 Design, Automation \& Test in Europe Conference \& Exhibition (DATE) (pp. 1599-1602). IEEE.

[9] Xing, S.; Qian, S.; Cheng, B.; Cao, J.; Xue, G.; Yu, J.; Li, M. (2019, July): A QoS-oriented Scheduling and Autoscaling Framework for Deep Learning. In 2019 International Joint Conference on Neural Networks (IJCNN) (pp. 1-8). IEEE.

[10] Koshimizu, T.; Gengtian, S.; Wang, H.; Pan, Z.; Liu, J.; Shimamoto, S. (2020). Multi-dimensional affinity propagation clustering applying a machine learning in 5G-cellular V2X. IEEE Access, 8, pp. 94560-94574.

[11] Yin, Y.; Cao, Z.; Xu, Y.; Gao, H.; Li, R.; Mai, Z. (2020): QoS Prediction for Service Recommendation With Features Learning in Mobile Edge Computing Environment. IEEE Transactions on Cognitive Communications and Networking, 6(4), 1136-1145.

[12] Aldhyani, T. H.; Alrasheedi, M.; Alqarni, A. A.; Alzahrani, M. Y.; Bamhdi, A. M. (2020): Intelligent hybrid model to enhance time series models for predicting network traffic. IEEE Access, 8, pp. 130431-130451.

[13] Robinson, Y. H.; Rajaram, M. (2015): Energy-aware multipath routing scheme based on particle swarm optimization in mobile ad hoc networks. The Scientific World Journal, 2015.

[14] Trivedi, M. C., \& Sharma, A. K. (2016): QoS Improvement in MANET using particle swarm optimization algorithm. In Proceedings of the International Congress on Information and Communication Technology (pp. 181-189). Springer, Singapore.

[15] Zhou, Y.; Wang, N.; Xiang, W. (2016): Clustering hierarchy protocol in wireless sensor networks using an improved PSO algorithm. IEEE access, 5, pp.2241-2253.

[16] Abdrabou, A.; Zhuang, W. (2006): A position-based QoS routing scheme for UWB mobile ad hoc networks. IEEE Journal on Selected Areas in Communications, 24(4), pp.850-856.

[17] Joshiand S.S.; BiradarS.R.; (2021): CDLN - Cluster Distance Based Data Forwarding And Optimal Leader Election Using Fuzzy Inference In Wireless Network, Indian Journal of Computer Science and Engineering, 12(4), pp.1074-1083.

[18] Nalluri S.; Kurra R.R. (2021): Uunsupervised Feature Selection For Text Clustering Using Differential Inverse Document Frequency, Journal of Computer Science and Engineering , 12(4), pp. 790-797.

[19] Chavan P.; Reddy S. (2021): Integrated Cross Layer Optimization Approach For Quality Of Service Enhancement in Wireless Network, Journal of Computer Science and Engineering, 12(4), pp. 885-898.

[20] Suchitha S.; Prasanthi B.G. (2021): Design of a Bio Based Approach For Congestion Control In Wireless Sensor Network, Journal of Computer Science and Engineering, 12(4), pp. 1065-1073.

\section{Authors Profile}
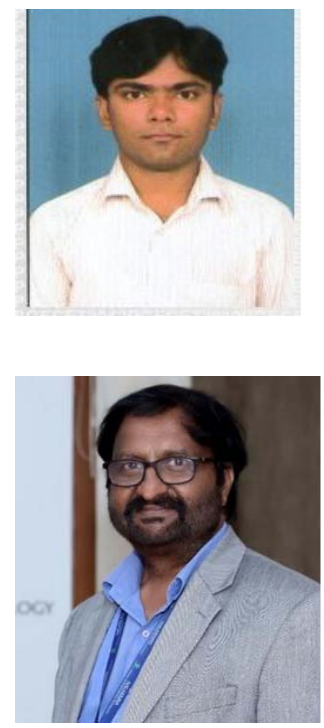

V. Surya Narayana Reddy is currently Pursuing Ph.D. in the Department of Computer Science at VTU, Belagavi under the Research Centre of NCET, Bangalore. He received his M.Tech degree in Computer Science from JNTUA, Anantapur in 2014. He has been working as an Assistant professor in VNRVJIET. His Research interests include in IDS in Network using latest Machine and Deep Learning techniques, Artificial Intelligence, Data science etc. Reviewed and Handled many Research papers on latest techniques and guided many students in the same technology.

Dr. Jitendranath Mungara, Principal \& Prof. is a dynamic, team spirited, and performance driven engineering professional and Educational Leader in the fields of Academic Administration, Research, Quality Assurance, Educational Consultancy and also Extension Activities. He completed many Technical and management Proficiency Certificate courses in USA. He is good in SQA \& TQM Audits. He taught many MOOC Courses to the faculty and students under the train the trainer's concept. He is author of over $150+$ scholarly research/ review papers, including $100+$ reputed and peer reviewed international journal (Scopus/SCI/UGC/IEEE/ Springer/WOS) papers with 310+ Citation index, 10+ h-index and $10+\mathrm{i} 10$ index. He has won several research paper awards in different National and International conferences and symposiums. He is double Ph.D. holder from different universities and has filed 6 patents and published 3 Patents. He is Member in many leading professional Societies and Forums. He is reviewer and editorial board member/ Advisory board for many reputed/ UGC approved International/ National Journals and has published 3 Technical books in the field of Computer Engineering. He delivered many Keynote Speeches and Chaired Technical sessions in many International, National Conferences and Symposiums. 